\title{
Discovery of new bright ULIRGs from the IRAS PSC/FSC Combined Catalogue
}

\author{
G. A. Mikayelyan, A. M. Mickaelian, H. V. Abrahamyan, G. M. Paronyan
}

NAS RA V. Ambartsumian Byurakan Astrophysical Observatory (BAO)

E-mail: gormick@mail.ru

\begin{abstract}
High-luminosity IR galaxies (LIRGs, ULIRGs, and HLIRGs) are important for studies related to star-formation processes in the early Universe, as their luminosity allows to detect them at large distances. High IR indicates active star-formation and often starburst processes, which is typical to HII (starburst, SB) and AGN. An interesting question is whether the starburst triggers $A G N$ or vice versa or there is no direct impact. Considering that very often such objects manifest double and multiple structure, it is also interesting to investigate the interrelationship between the SB, nuclear activity and interactions or merging. We have analyzed the IRAS PSC/FSC Combined Catalogue for search for new bright ULIRGs. By means of the SDSS DR14 data, namely redshifts for those objects having spectroscopy, we have calculated the IR luminosities and have found 114 very high-luminosity IR galaxies; 107 ULIRGs and 7 HLIRGs. Among them, 48 new ULIRGs and 7 new HLIRGs have been discovered. These objects have been studied by SDSS color-color, luminosity-redshift and other diagrams. Further studies will include the content of the sample for activity types and other available data.
\end{abstract}

Keywords: IR sources, IR galaxies, starburst galaxies, AGN, ULIRGs, HLIRGs, interacting galaxies, mergers.

\section{Introduction}

The InfraRed Astronomical Satellite (IRAS) was the first-ever space telescope to perform a survey of the entire night sky at infrared wavelengths. Launched on 25 January 1983, its mission lasted ten months. The telescope was a joint project of the United States(NASA), the Netherlands (NIVR), and the United Kingdom(SERC). It mapped $96 \%$ of the sky four times, at 12, 25, 60 and 100 micrometers, with resolutions ranging from 30 arcseconds at 12 micrometers to 2 arcminutes at 100 micrometers. It discovered about 350,000 sources given in several catalogues.

One of the most important revelations of the extragalactic observations in near- , mid- , and far- IR (NIR, MIR, and FIR) was the discovery of IR galaxies. These galaxies emit the most part of their energy in IR. The main part of galaxies that have 
powerful IR emission are AGN and Starburst (SB) galaxies. David Sanders and Felix Mirabel suggested in 1996 to split high-luminosity IR galaxies into three main classes (Sanders \& Mirabel 1996): galaxies with $10^{11} \mathrm{~L}_{\odot}<\mathrm{L}_{\mathrm{IR}}<10^{12} \mathrm{~L}_{\odot}$ were named $\mathrm{LIRGs}$ (Luminous InfraRed Galaxies), galaxies with $10^{12} \mathrm{~L} \odot_{\odot}<\mathrm{L}_{\mathbb{R}}<10^{13} \mathrm{~L} \odot_{\odot}$ were named ULIRGs (Ultra-Luminous InfraRed Galaxies) and galaxies with $10^{13} \mathrm{~L}_{\odot}<\mathrm{L}_{\mathrm{I}}$ were named HLIRGs (Hyper-Luminous InfraRed Galaxies).Sylvain Veilleux (Veilleux 2002) showed that the percent of AGNs increases parallel to IR luminosity, so among the sources that have the highest IR luminosities there are full of QSOs and Seyfert galaxies, and among the sources that have lower IR luminosities there are mostly Starburst galaxies.

The IRAS PSC/FSC Combined Catalogue contains 345,163 sources (Abrahamyan et al. 2015). The advantage against more recent catalogues is that in this catalogue FIR fluxes are also given; the sources are available in the whole sky and it is homogeneous material for extragalactic research. The coordinates of the sources of IRAS PSC/FSC Combined Catalogue have been improved by other catalogues, in particular by AKARI-IRC (Ishihara et al. 2010), AKARI-FIS (Yamamura et al. 2010) and WISE (Cutri et al. 2013) and most of the sources have accuracy of better than 1". For the first time it gives an opportunity to study IRAS sources using most recent accurate astrometry, photometry, as well as SDSS spectroscopy. We have benefited from these accurate data to correctly identify optical counterparts and select those objects having SDSS data.

\section{Sample selection}

From the IRAS PSC/FSC Combined Catalogue, we have taken sources that were checked by WISE considering that the accuracy of the WISE coordinates is significantly higher $\left(<1^{\prime \prime}\right)$ than the coordinates of the two other AKARI catalogues.

The selected sample was cross-correlated with SDSS DR14 (Abolfathi et al. 2017). Considering that the accuracy of WISE coordinates is $\sim 1^{\prime \prime}$, and the accuracy of SDSS coordinates is $\sim 0.1^{\prime \prime}$, the cumulative accuracy will be:

$$
\begin{gathered}
\sigma=\sqrt{\sigma_{W I S E}^{2}+\sigma_{S D S S}^{2}} \\
\sigma=\sqrt{1^{2}+0.1^{2}} \approx 1.005
\end{gathered}
$$

Corresponding $3 \sigma$ was taken as the search radius. Only the SDSS objects that had spectroscopical data and were the only optical counterparts in the radius of $3 \sigma$ were taken from the results of cross-correlation. This way 6,346 objects were found; galaxies brighter than $17.77^{\mathrm{m}}$. 


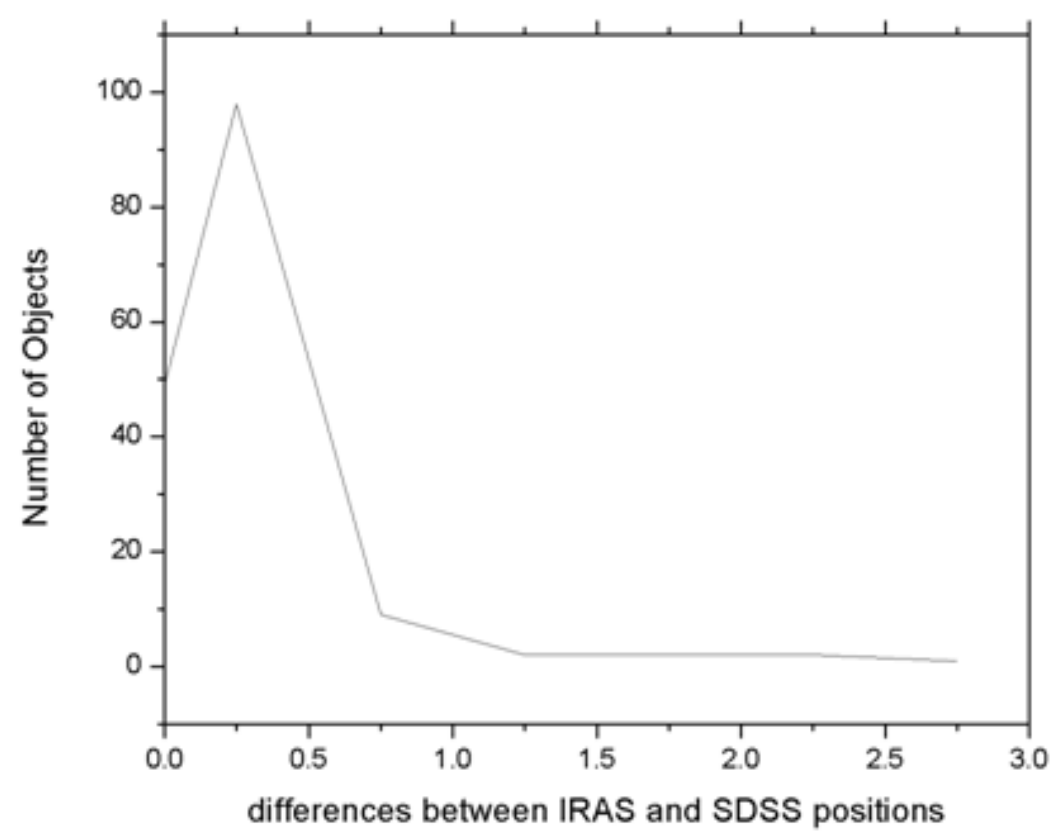

Figure 1. RA and DEC differences between IRAS and SDSS positions. Most of the associations are within $1 "$.

\section{IR luminosities and ULIRGs}

For the calculation of the IR luminosities of the sources the flux densities for each source in 12, 25, 60 and $120 \mu$ bands that are given in IRAS PSC/FSC Combined Catalogue are necessary. Therefore, from the 6,346 objects only those were chosen that had the data on the flux densities in all four bands. Only 6,270 sources had such data. The IR and FIR luminosities were calculated by the following formulae (Duc et al. 1997):

$$
\begin{gathered}
L_{\text {ir }}=5.6 \times 10^{5} R^{2}\left(13.56 f_{12}+5.26 f_{25}+2.54 f_{60}+f_{100}\right) L_{\odot} \\
L_{\text {fir }}=5.6 \times 10^{5} R^{2}\left(2.58 f_{60}+f_{100}\right) L_{\odot}
\end{gathered}
$$

where the $R$ is the distance of the object in $M p c$ and $f_{12}, f_{25}, f_{60}, f_{100}$ are the flux densities given in IRAS PSC/FSC Combined Catalogue in 12, 25, 60, 100 $\mu$ bands, respectively. Out of 6,270 objects only those were selected having IR luminosities greater than $10^{12} L_{\odot}$ (the lower limit of ULIRG's luminosity) and having stellar magnitudes in $r$ band greater than $17.77^{\mathrm{m}}$ (the spectroscopic limit for SDSS galaxies).

As a result, 114 extragalactic objects were selected that have high IR luminosity and correspond to ULIRG/HLIRG classes. 


\section{Results}

In Table 1, we give the list of 114 ULIRGs/HLIRGs found from IRAS PSC/FSC Combined Catalogue.

Table 1. The list of 114 ULIRGs/HLIRGs found from IRAS PSC/FSC Combined

Catalogue based on SDSS redshifts; Byurakan ULIRGs.

\begin{tabular}{|c|c|c|c|c|c|c|c|c|c|c|c|c|c|c|}
\hline & \multicolumn{2}{|c|}{ IRAS coordinates } & & \multicolumn{4}{|c|}{ IRAS fluxes (mJy) } & \multicolumn{5}{|c|}{ SDSS model magnitudes } & \multirow[b]{2}{*}{$\mathbf{z}$} & \multirow[b]{2}{*}{$\begin{array}{c}\text { LOG } \\
\text { (Lir/ } \\
\left.\mathrm{L}_{\text {sun }}\right)\end{array}$} \\
\hline$\#$ & RA J2000 & DEC J2000 & $\begin{array}{c}r \\
(")\end{array}$ & $\mathbf{F 1 2}$ & $\mathbf{F} 25$ & F60 & F100 & $\begin{array}{c}\mathbf{u} \\
(\mathbf{m a g})\end{array}$ & $\begin{array}{c}\mathbf{g} \\
\text { (mag) }\end{array}$ & $\begin{array}{c}\mathbf{r} \\
(\mathrm{mag})\end{array}$ & $\begin{array}{c}\mathrm{i} \\
(\mathrm{mag})\end{array}$ & $\begin{array}{c}z \\
\text { (mag) }\end{array}$ & & \\
\hline 1 & 002000.270 & +320802.82 & 0.19 & 0.29 & 0.19 & 0.33 & 4.76 & 18.97 & 17.53 & 16.70 & 16.16 & 15.87 & 0.110 & 12.059 \\
\hline 2 & 003025.608 & -092617.39 & 1.29 & 1.03 & 1.61 & 11.40 & 47.70 & 19.93 & 18.46 & 17.74 & 17.31 & 17.04 & 0.129 & 13.157 \\
\hline 3 & 003321.837 & -050544.63 & 2.42 & 0.46 & 5.66 & 27.60 & 99.80 & 19.31 & 17.12 & 16.12 & 15.65 & 15.27 & 0.053 & 12.740 \\
\hline 4 & 004459.123 & +192141.45 & 0.26 & 0.14 & 0.18 & 0.32 & 0.82 & 16.91 & 16.78 & 16.65 & 16.36 & 16.38 & 0.179 & 12.073 \\
\hline 5 & 011427.481 & +272138.98 & 1.33 & 1.34 & 0.76 & 15.60 & 115.00 & 19.33 & 18.07 & 17.16 & 16.55 & 16.13 & 0.109 & 13.277 \\
\hline 6 & 011956.727 & +133431.51 & 0.29 & 0.10 & 0.16 & 0.31 & 0.80 & 19.23 & 18.00 & 17.29 & 16.92 & 16.68 & 0.191 & 12.052 \\
\hline 7 & 012709.340 & +084015.52 & 2.84 & 0.57 & 0.34 & 2.64 & 39.00 & 19.59 & 17.35 & 16.34 & 15.80 & 15.36 & 0.045 & 12.026 \\
\hline 8 & 013758.267 & -083430.44 & 0.04 & 0.16 & 0.18 & 0.29 & 0.76 & 20.43 & 18.39 & 17.38 & 16.77 & 16.41 & 0.189 & 12.128 \\
\hline 9 & 015950.248 & +002340.98 & 0.12 & 0.12 & 0.54 & 2.22 & 2.16 & 15.93 & 15.70 & 15.52 & 15.26 & 15.30 & 0.163 & 12.444 \\
\hline 10 & 020422.321 & -093757.65 & 0.46 & 0.15 & 0.17 & 0.34 & 0.76 & 19.77 & 18.40 & 17.63 & 17.04 & 16.82 & 0.192 & 12.133 \\
\hline 11 & 022423.087 & -021030.55 & 0.23 & 0.12 & 0.16 & 0.30 & 0.97 & 20.82 & 19.01 & 17.63 & 17.06 & 16.70 & 0.173 & 12.015 \\
\hline 12 & 022840.542 & -093505.68 & 2.02 & 0.86 & 0.88 & 5.87 & 80.10 & 17.52 & 16.58 & 16.18 & 15.79 & 15.63 & 0.068 & 12.682 \\
\hline 13 & 024417.066 & -003038.22 & 0.26 & 0.11 & 0.16 & 0.48 & 0.83 & 21.51 & 19.00 & 17.65 & 17.11 & 16.68 & 0.200 & 12.147 \\
\hline 14 & 032322.858 & -075615.21 & 0.16 & 0.10 & 0.13 & 1.00 & 1.69 & 18.95 & 18.00 & 17.37 & 16.93 & 16.67 & 0.166 & 12.166 \\
\hline 15 & 032902.594 & -005539.10 & 0.39 & 0.46 & 0.54 & 1.78 & 12.80 & 17.30 & 15.83 & 15.04 & 14.61 & 14.33 & 0.067 & 12.043 \\
\hline 16 & 073735.222 & +353621.36 & 0.32 & 0.12 & 0.15 & 1.88 & 4.45 & 15.10 & 13.86 & 13.11 & 12.72 & 12.40 & 0.819 & 13.523 \\
\hline 17 & 080407.412 & +391927.69 & 0.20 & 0.23 & 0.13 & 0.27 & 0.73 & 19.73 & 18.38 & 17.54 & 17.05 & 16.76 & 0.163 & 12.064 \\
\hline 18 & 081331.289 & +254503.15 & 0.08 & 0.11 & 0.15 & 0.28 & 0.54 & 16.42 & 16.00 & 15.61 & 15.27 & 15.23 & 1.510 & 13.259 \\
\hline 19 & 082445.643 & +153943.83 & 0.30 & 0.13 & 0.18 & 0.36 & 0.80 & 20.11 & 18.64 & 17.69 & 17.21 & 16.94 & 0.220 & 12.229 \\
\hline 20 & 083148.881 & +04 2939.07 & 0.05 & 0.17 & 0.26 & 0.43 & 0.77 & 16.34 & 15.80 & 15.35 & 15.05 & 14.78 & 0.174 & 12.147 \\
\hline 21 & 083457.652 & +484515.63 & 0.19 & 0.09 & 0.09 & 0.34 & 2.43 & 19.19 & 17.60 & 16.70 & 16.21 & 15.93 & 0.175 & 12.101 \\
\hline 22 & 085448.878 & +200630.75 & 0.11 & 0.28 & 0.43 & 0.89 & 1.16 & 16.57 & 15.93 & 15.42 & 15.04 & 14.71 & 0.778 & 13.411 \\
\hline 23 & 085517.068 & +130509.65 & 0.22 & 0.11 & 0.21 & 0.24 & 1.63 & 19.32 & 18.13 & 17.40 & 16.90 & 16.65 & 0.157 & 12.002 \\
\hline 24 & 085706.358 & +190853.69 & 0.19 & 0.11 & 0.12 & 0.33 & 0.52 & 17.25 & 17.19 & 16.99 & 17.12 & 16.61 & 0.331 & 12.440 \\
\hline 25 & 085841.758 & +104122.04 & 0.16 & 0.11 & 0.19 & 1.12 & 1.95 & 18.93 & 17.49 & 16.62 & 16.08 & 15.81 & 0.148 & 12.134 \\
\hline 26 & 085923.600 & +473610.53 & 0.12 & 0.09 & 0.12 & 0.44 & 0.88 & 20.80 & 18.76 & 17.60 & 17.02 & 16.69 & 0.180 & 12.024 \\
\hline 27 & 090025.376 & +390354.38 & 0.65 & 0.32 & 1.70 & 7.43 & 4.59 & 18.72 & 17.34 & 16.85 & 16.50 & 16.25 & 0.058 & 12.069 \\
\hline 28 & 090554.723 & +241829.03 & 0.32 & 0.05 & 0.09 & 0.36 & 0.59 & 19.98 & 18.61 & 17.72 & 17.24 & 16.87 & 0.232 & 12.057 \\
\hline 29 & 091438.146 & +093322.95 & 0.12 & 0.11 & 0.15 & 0.28 & 1.19 & 19.54 & 18.27 & 17.26 & 16.78 & 16.43 & 0.246 & 12.300 \\
\hline 30 & 092159.400 & +450912.61 & 0.22 & 0.06 & 0.13 & 0.23 & 0.57 & 18.58 & 18.05 & 17.73 & 17.46 & 17.34 & 0.235 & 12.054 \\
\hline 31 & 092608.518 & +282402.59 & 0.15 & 0.08 & 0.21 & 0.25 & 0.62 & 19.19 & 18.24 & 17.62 & 17.17 & 17.00 & 0.203 & 12.062 \\
\hline 32 & 095846.856 & +505456.54 & 0.06 & 0.05 & 0.06 & 0.22 & 1.37 & 19.57 & 18.10 & 17.25 & 16.80 & 16.56 & 0.214 & 12.042 \\
\hline 33 & 100149.527 & +284709.01 & 0.10 & 0.16 & 0.26 & 0.23 & 0.34 & 18.54 & 17.96 & 16.83 & 16.63 & 16.81 & 0.185 & 12.100 \\
\hline 34 & 100532.126 & +305318.27 & 0.14 & 0.09 & 0.10 & 0.20 & 0.63 & 18.89 & 17.81 & 17.07 & 16.61 & 16.35 & 0.234 & 12.099 \\
\hline 35 & 100541.874 & +433240.60 & 0.25 & 0.13 & 0.19 & 0.56 & 0.87 & 16.39 & 16.38 & 16.38 & 16.18 & 16.28 & 0.179 & 12.122 \\
\hline 36 & 102617.480 & +303643.24 & 0.19 & 0.06 & 0.16 & 0.26 & 0.67 & 18.75 & 18.04 & 17.54 & 17.40 & 16.87 & 0.340 & 12.380 \\
\hline 37 & 103343.863 & +621106.27 & 0.56 & 0.08 & 0.08 & 0.20 & 0.57 & 19.76 & 18.49 & 17.57 & 17.12 & 16.84 & 0.229 & 12.038 \\
\hline 38 & 103359.475 & +355509.18 & 0.14 & 0.09 & 0.16 & 0.52 & 0.79 & 17.10 & 16.97 & 16.66 & 16.35 & 16.28 & 0.169 & 12.002 \\
\hline 39 & 104000.549 & +485744.44 & 0.19 & 0.08 & 0.11 & 0.64 & 0.66 & 19.16 & 18.34 & 17.76 & 17.27 & 17.20 & 0.176 & 12.013 \\
\hline 40 & 105223.573 & +440847.17 & 0.73 & 0.12 & 0.17 & 3.53 & 5.41 & 19.37 & 17.59 & 16.83 & 16.32 & 16.04 & 0.092 & 12.114 \\
\hline 41 & 105330.955 & +331342.39 & 0.14 & 0.11 & 0.13 & 0.21 & 0.54 & 19.77 & 18.56 & 17.63 & 17.20 & 16.90 & 0.244 & 12.170 \\
\hline 42 & 105839.325 & +382906.77 & 0.40 & 0.05 & 0.09 & 0.62 & 0.75 & 19.78 & 18.35 & 17.53 & 17.10 & 16.83 & 0.208 & 12.086 \\
\hline 43 & 110832.952 & +40 3731.72 & 0.07 & 0.10 & 0.13 & 0.68 & 1.02 & 19.39 & 18.07 & 17.36 & 16.92 & 16.66 & 0.166 & 12.045 \\
\hline 44 & 111211.118 & +273256.18 & 0.64 & 0.07 & 0.25 & 0.25 & 0.83 & 18.46 & 17.82 & 17.42 & 17.13 & 17.04 & 0.235 & 12.210 \\
\hline 45 & 111438.892 & +324133.60 & 0.10 & 0.17 & 0.35 & 1.59 & 1.52 & 21.22 & 18.94 & 17.13 & 16.09 & 15.80 & 0.188 & 12.447 \\
\hline
\end{tabular}




\begin{tabular}{|c|c|c|c|c|c|c|c|c|c|c|c|c|c|c|}
\hline 46 & 111603.133 & +020852.30 & 0.04 & 0.17 & 0.19 & 0.40 & 0.33 & 16.81 & 16.83 & 16.68 & 16.44 & 16.50 & 0.211 & 12.219 \\
\hline 47 & 112114.226 & +032546.82 & 0.07 & 0.17 & 0.22 & 0.36 & 1.16 & 17.46 & 17.29 & 17.05 & 16.66 & 16.66 & 0.152 & 12.039 \\
\hline 48 & 112233.084 & +501527.98 & 0.21 & 0.09 & 0.12 & 0.19 & 0.95 & 19.98 & 18.52 & 17.69 & 17.12 & 16.96 & 0.195 & 12.010 \\
\hline 49 & 114122.043 & +405950.39 & 0.16 & 0.20 & 0.14 & 1.02 & 1.51 & 19.99 & 18.33 & 17.42 & 16.89 & 16.55 & 0.149 & 12.156 \\
\hline 50 & 114430.922 & +254634.98 & 0.38 & 0.15 & 0.15 & 0.29 & 0.65 & 18.64 & 17.44 & 16.56 & 16.12 & 15.84 & 0.185 & 12.082 \\
\hline 51 & 115314.237 & +131427.74 & 0.19 & 0.10 & 0.30 & 2.58 & 3.32 & 19.88 & 18.30 & 17.59 & 17.04 & 16.82 & 0.127 & 12.259 \\
\hline 52 & 115744.359 & +120750.97 & 0.26 & 0.17 & 0.13 & 0.22 & 0.55 & 18.78 & 17.57 & 16.78 & 16.35 & 16.06 & 0.183 & 12.048 \\
\hline 53 & 115753.218 & -031537.03 & 0.21 & 0.12 & 0.30 & 0.33 & 0.63 & 17.91 & 17.74 & 17.20 & 16.69 & 16.82 & 0.214 & 12.231 \\
\hline 54 & 115816.724 & +132624.23 & 0.09 & 0.10 & 0.16 & 0.23 & 0.53 & 17.95 & 17.59 & 17.40 & 17.26 & 17.12 & 0.439 & 12.606 \\
\hline 55 & 120116.658 & +233452.85 & 0.34 & 0.12 & 0.14 & 0.26 & 0.60 & 19.19 & 18.28 & 17.53 & 17.22 & 16.90 & 0.263 & 12.281 \\
\hline 56 & 120205.612 & +112812.13 & 0.29 & 0.11 & 0.16 & 0.95 & 1.15 & 18.74 & 17.93 & 17.43 & 17.03 & 16.93 & 0.194 & 12.253 \\
\hline 57 & 120226.765 & -012915.35 & 0.13 & 0.36 & 0.54 & 2.41 & 2.72 & 17.28 & 16.96 & 16.62 & 16.27 & 16.10 & 0.150 & 12.506 \\
\hline 58 & 120424.554 & +192509.99 & 0.32 & 0.11 & 0.37 & 1.76 & 1.78 & 19.50 & 18.15 & 17.46 & 17.02 & 16.79 & 0.168 & 12.360 \\
\hline 59 & 120526.443 & +22 4337.56 & 0.15 & 0.62 & 0.54 & 0.51 & 21.00 & 19.42 & 17.78 & 16.77 & 16.16 & 15.74 & 0.076 & 12.250 \\
\hline 60 & 121119.098 & +055523.85 & 0.21 & 0.15 & 0.17 & 0.24 & 0.55 & 19.67 & 18.38 & 17.53 & 16.98 & 16.72 & 0.201 & 12.128 \\
\hline 61 & 121509.822 & +092709.37 & 0.27 & 0.09 & 0.27 & 0.21 & 0.45 & 20.15 & 18.66 & 17.61 & 17.17 & 16.90 & 0.263 & 12.286 \\
\hline 62 & 122906.906 & +322417.75 & 0.30 & 0.09 & 0.15 & 0.57 & 0.76 & 19.98 & 17.79 & 17.05 & 16.64 & 16.40 & 0.173 & 12.022 \\
\hline 63 & 123121.374 & +275523.93 & 0.10 & 0.08 & 0.13 & 0.35 & 0.67 & 20.18 & 18.41 & 17.44 & 16.96 & 16.69 & 0.212 & 12.090 \\
\hline 64 & 123716.002 & +040148.67 & 0.57 & 0.10 & 0.32 & 0.66 & 1.03 & 19.18 & 17.67 & 16.81 & 16.30 & 15.95 & 0.146 & 12.025 \\
\hline 65 & 123820.209 & +1750 39.22 & 0.16 & 0.14 & 0.13 & 0.26 & 0.39 & 17.22 & 16.61 & 16.43 & 16.29 & 16.24 & 0.452 & 12.658 \\
\hline 66 & 124526.988 & +652358.40 & 0.05 & 0.06 & 0.07 & 0.21 & 0.65 & 17.56 & 17.48 & 17.30 & 17.36 & 16.95 & 0.320 & 12.235 \\
\hline 67 & 125100.438 & +660326.87 & 0.07 & 0.06 & 0.07 & 0.17 & 0.58 & 16.78 & 16.75 & 16.62 & 16.79 & 16.41 & 0.282 & 12.117 \\
\hline 68 & 125120.052 & +02 1902.43 & 0.25 & 0.09 & 0.13 & 0.47 & 0.89 & 19.40 & 18.42 & 17.58 & 17.18 & 16.82 & 0.253 & 12.290 \\
\hline 69 & 125410.444 & +035951.70 & 0.20 & 0.12 & 0.29 & 0.25 & 0.82 & 19.65 & 18.30 & 17.47 & 16.89 & 16.58 & 0.168 & 12.032 \\
\hline 70 & 125604.931 & +023831.96 & 0.22 & 0.11 & 0.24 & 0.29 & 1.49 & 19.81 & 18.45 & 17.37 & 16.85 & 16.50 & 0.219 & 12.272 \\
\hline 71 & 125739.331 & +08 0931.74 & 0.04 & 0.09 & 0.12 & 0.59 & 0.78 & 17.76 & 17.47 & 17.17 & 17.24 & 16.91 & 0.272 & 12.362 \\
\hline 72 & 130118.171 & +110601.88 & 0.09 & 0.12 & 0.14 & 0.29 & 0.83 & 19.97 & 18.70 & 17.55 & 17.06 & 16.88 & 0.174 & 12.002 \\
\hline 73 & 130618.579 & +085241.43 & 0.21 & 0.13 & 0.26 & 0.29 & 0.62 & 17.78 & 17.55 & 17.29 & 16.92 & 16.95 & 0.172 & 12.041 \\
\hline 74 & 132211.965 & +013034.31 & 0.17 & 0.10 & 0.32 & 0.33 & 0.72 & 18.13 & 17.74 & 17.37 & 17.22 & 17.59 & 0.184 & 12.114 \\
\hline 75 & 132244.041 & +633725.16 & 0.58 & 0.06 & 0.07 & 0.59 & 0.91 & 19.85 & 18.41 & 17.50 & 17.04 & 16.83 & 0.200 & 12.064 \\
\hline 76 & 132419.895 & +053704.79 & 0.15 & 0.26 & 0.40 & 1.17 & 0.71 & 18.73 & 18.25 & 17.55 & 17.04 & 16.98 & 0.203 & 12.494 \\
\hline 77 & 133715.938 & +030936.79 & 0.33 & 0.11 & 0.16 & 0.22 & 0.69 & 19.11 & 18.12 & 17.33 & 16.81 & 16.56 & 0.192 & 12.035 \\
\hline 78 & 134442.108 & +55 5313.31 & 0.53 & 0.24 & 2.28 & 21.70 & 21.40 & 16.59 & 14.99 & 14.31 & 13.84 & 13.71 & 0.037 & 12.090 \\
\hline 79 & 134733.379 & +121724.35 & 0.30 & 0.14 & 0.67 & 1.92 & 2.06 & 18.16 & 16.37 & 15.35 & 14.79 & 14.59 & 0.120 & 12.201 \\
\hline 80 & 134840.199 & +581852.16 & 0.96 & 0.05 & 0.07 & 1.27 & 1.73 & 19.90 & 18.44 & 17.57 & 17.10 & 16.78 & 0.158 & 12.105 \\
\hline 81 & 135043.182 & +372434.11 & 0.18 & 0.11 & 0.09 & 0.30 & 0.94 & 19.46 & 17.97 & 17.00 & 16.60 & 16.30 & 0.248 & 12.239 \\
\hline 82 & 135550.208 & +204614.67 & 0.15 & 0.19 & 0.21 & 0.26 & 0.54 & 16.16 & 15.94 & 15.66 & 15.31 & 15.48 & 0.197 & 12.184 \\
\hline 83 & 140438.810 & +432707.52 & 0.11 & 0.12 & 0.29 & 0.62 & 0.99 & 16.18 & 15.63 & 15.30 & 15.33 & 14.92 & 0.323 & 12.625 \\
\hline 84 & 141842.223 & +423343.39 & 0.02 & 0.10 & 0.09 & 0.31 & 0.57 & 16.54 & 16.40 & 16.43 & 16.44 & 16.37 & 0.420 & 12.556 \\
\hline 85 & 142231.386 & +260205.42 & 0.39 & 0.18 & 0.15 & 1.49 & 1.99 & 18.28 & 17.49 & 17.08 & 16.70 & 16.54 & 0.159 & 12.287 \\
\hline 86 & 142516.043 & +162307.97 & 0.16 & 0.10 & 0.25 & 0.54 & 0.98 & 19.47 & 17.76 & 16.89 & 16.47 & 16.25 & 0.185 & 12.154 \\
\hline 87 & 142727.266 & -005841.16 & 0.19 & 0.12 & 0.32 & 0.91 & 1.28 & 20.05 & 18.44 & 17.60 & 17.05 & 16.69 & 0.162 & 12.183 \\
\hline 88 & 143535.554 & +012834.73 & 0.18 & 0.13 & 0.22 & 0.35 & 0.65 & 20.10 & 18.70 & 17.77 & 17.33 & 17.00 & 0.232 & 12.281 \\
\hline 89 & 144012.775 & +615633.19 & 0.20 & 0.06 & 0.09 & 0.32 & 0.68 & 17.20 & 17.01 & 16.83 & 16.97 & 16.55 & 0.276 & 12.198 \\
\hline 90 & 144104.407 & +532008.94 & 0.33 & 0.07 & 0.35 & 1.95 & 2.39 & 18.27 & 17.08 & 16.47 & 15.75 & 15.72 & 0.105 & 12.002 \\
\hline 91 & 144920.716 & +42 2101.45 & 0.23 & 0.11 & 0.23 & 0.40 & 0.56 & 19.10 & 18.05 & 17.28 & 16.95 & 16.81 & 0.179 & 12.063 \\
\hline 92 & 145054.177 & +350837.94 & 0.17 & 0.07 & 0.09 & 0.64 & 1.21 & 18.48 & 17.57 & 16.91 & 16.38 & 16.19 & 0.206 & 12.162 \\
\hline 93 & 145549.433 & +485436.34 & 0.08 & 0.08 & 0.20 & 0.53 & 0.54 & 18.82 & 18.22 & 17.36 & 17.28 & 17.07 & 0.247 & 12.281 \\
\hline 94 & 150259.016 & +034004.00 & 0.16 & 0.09 & 0.08 & 0.23 & 1.09 & 24.25 & 18.30 & 17.49 & 17.05 & 16.83 & 0.218 & 12.098 \\
\hline 95 & 150539.529 & +574307.33 & 0.30 & 0.12 & 0.07 & 1.02 & 1.50 & 19.07 & 18.30 & 17.76 & 17.31 & 17.18 & 0.151 & 12.075 \\
\hline 96 & 151318.050 & +244655.40 & 0.19 & 0.06 & 0.17 & 0.52 & 0.56 & 19.19 & 18.07 & 17.13 & 16.61 & 16.34 & 0.220 & 12.134 \\
\hline 97 & 152238.091 & +33 3136.10 & 0.25 & 0.08 & 0.35 & 1.77 & 1.89 & 17.31 & 16.79 & 16.45 & 15.89 & 16.06 & 0.125 & 12.109 \\
\hline 98 & 152443.874 & +234010.41 & 0.20 & 0.07 & 0.18 & 1.30 & 1.48 & 19.31 & 17.94 & 17.11 & 16.58 & 16.32 & 0.139 & 12.045 \\
\hline 99 & 153457.252 & +233011.56 & 1.79 & 0.48 & 7.91 & 104.00 & 112.00 & 15.63 & 13.96 & 13.08 & 12.61 & 12.29 & 0.018 & 12.149 \\
\hline 100 & 154316.002 & +14 0731.93 & 0.21 & 0.07 & 0.10 & 0.20 & 1.11 & 19.19 & 18.24 & 17.58 & 17.18 & 16.94 & 0.221 & 12.080 \\
\hline 101 & 154351.941 & +162422.26 & 0.21 & 0.08 & 0.12 & 0.23 & 0.55 & 16.93 & 16.45 & 16.25 & 16.12 & 15.90 & 0.848 & 12.924 \\
\hline 102 & 154530.246 & +48 4608.97 & 0.09 & 0.07 & 0.11 & 0.28 & 0.45 & 16.43 & 16.32 & 16.30 & 16.30 & 16.06 & 0.400 & 12.460 \\
\hline 103 & 161849.253 & +442517.28 & 0.07 & 0.05 & 0.11 & 0.28 & 0.50 & 17.34 & 16.98 & 16.64 & 16.67 & 16.15 & 0.335 & 12.301 \\
\hline 104 & 163221.419 & +155145.77 & 0.64 & 0.07 & 0.12 & 1.48 & 1.99 & 19.67 & 18.52 & 17.70 & 17.26 & 16.96 & 0.242 & 12.527 \\
\hline 105 & 164258.823 & +394837.07 & 0.17 & 0.11 & 0.29 & 0.60 & 1.38 & 16.16 & 15.68 & 15.39 & 15.10 & 14.86 & 0.594 & 13.047 \\
\hline 106 & 170441.377 & +604430.56 & 0.07 & 0.07 & 0.13 & 0.30 & 0.70 & 15.60 & 15.43 & 15.25 & 15.19 & 14.82 & 0.372 & 12.458 \\
\hline
\end{tabular}




\begin{tabular}{|c|c|c|c|c|c|c|c|c|c|c|c|c|c|c|}
\hline 107 & 205519.286 & +003226.16 & 0.40 & 1.53 & 1.82 & 44.00 & 58.90 & 16.58 & 15.15 & 14.42 & 14.04 & 13.74 & 0.023 & 12.023 \\
\hline 108 & 214833.443 & +113147.78 & 0.03 & 0.05 & 0.07 & 0.45 & 1.80 & 19.93 & 18.13 & 17.19 & 16.69 & 16.37 & 5.225 & 13.556 \\
\hline 109 & 215236.547 & -074209.35 & 0.26 & 0.35 & 0.47 & 3.77 & 82.70 & 17.13 & 15.54 & 14.77 & 14.35 & 14.03 & 0.035 & 12.071 \\
\hline 110 & 223606.403 & +121843.84 & 0.20 & 0.38 & 0.53 & 3.57 & 49.10 & 18.57 & 17.25 & 16.54 & 16.09 & 15.83 & 0.071 & 12.492 \\
\hline 111 & 225302.457 & +172018.57 & 0.16 & 0.10 & 0.14 & 0.58 & 2.36 & 19.62 & 18.07 & 17.13 & 16.52 & 16.27 & 0.179 & 12.196 \\
\hline 112 & 225832.466 & +074940.59 & 0.20 & 0.12 & 0.22 & 0.61 & 1.14 & 18.76 & 18.00 & 17.58 & 17.40 & 16.99 & 0.396 & 12.755 \\
\hline 113 & 230833.953 & +052129.87 & 0.10 & 0.20 & 0.43 & 1.15 & 0.83 & 18.38 & 17.42 & 16.53 & 15.91 & 15.51 & 0.173 & 12.335 \\
\hline 114 & 235613.870 & -003210.00 & 1.51 & 0.14 & 0.12 & 0.24 & 0.59 & 19.19 & 16.90 & 16.07 & 15.68 & 15.38 & 0.189 & 12.041 \\
\hline
\end{tabular}

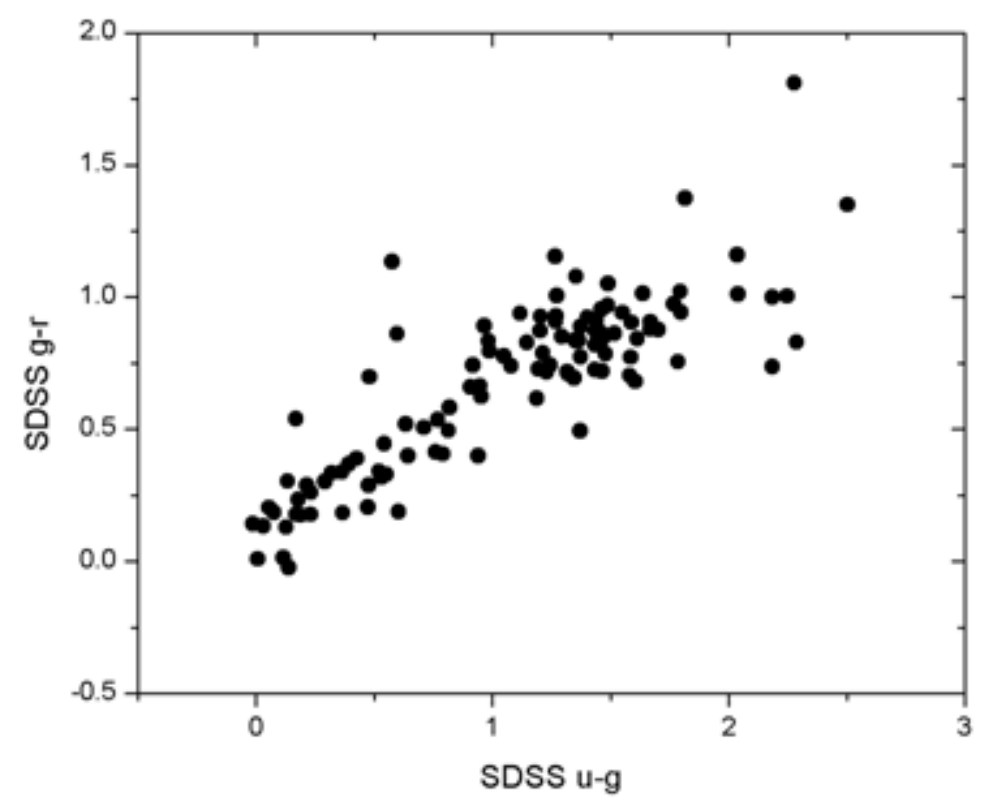

Figure 2. SDSS color-color diagram for 114 newly revealed ULIRGs and HLIRGs.

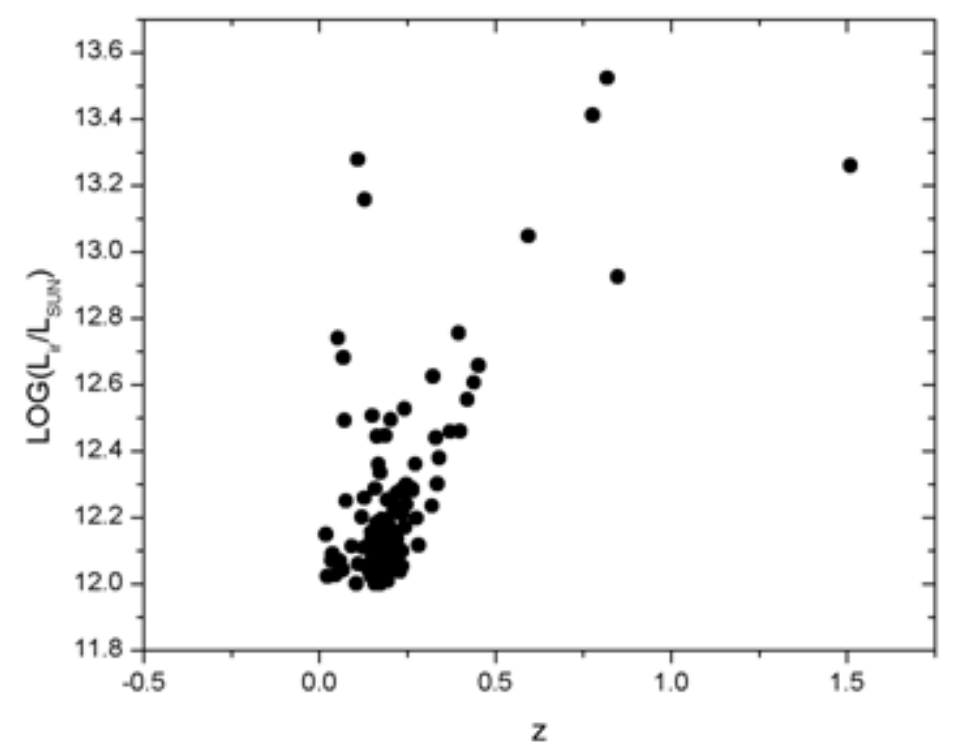

Figure 3. IR luminosity vs. redshift for 114 newly revealed ULIRGs and HLIRGs. The distribution of ULIRGs shows almost linear dependence in the range of Log $L_{i r} 12.0-12.8$ and $z$ 0.0-0.5. Higher redshifts are probably excluded by magnitude limit $\left(17.77^{\mathrm{m}}\right)$. Due to small numbers, the distribution of HLIRGs is not homogeneous. 
As a result, the objects were classified as 107 ULIRGs and 7 HLIRGs. The objects were checked by NED (The NASA/IPAC Extragalactic Database);out of 107 ULIRGs only 59 were known before, and 2 out of 7 HLIRGs were classified as ULIRGs. As a result, 48 new ULIRGs and 7 new HLIRGs were discovered. Considering homogeneity of the sample and the prevalence on the whole sky, it is significant quantity to make statistical study on such objects.

\section{Summary and Conclusion}

We have selected sources from the IRAS PSC/FSC Combined Catalogue to reveal new high-luminosity IR galaxies (LIRGs, ULIRGs, HLIRGs). We benefited from the improved coordinates of this catalog to accurately identify the optical counterparts, namely objects from SDSS classified as galaxies. As a result, 6,346 objects were selected having confident optical counterparts. Objects having SDSS spectroscopy (Petrosian magnitudes brighter than $17.77^{\mathrm{m}}$ ) were considered as the main sample to calculate their distances and luminosities. 114 objects were selected, including 107 ULIRGs and 7 HLIRGs. Among them, 48 ULIRGs and 7 HLIRGs are newly discovered ones. We call these objects Byurakan ULIRGs.

Considering the number of ULIRGs at different available (bright) magnitudes, one can extrapolate and conclude what is the real number of ULIRGs at fainter magnitudes, thus estimating their abundance in the Universe.

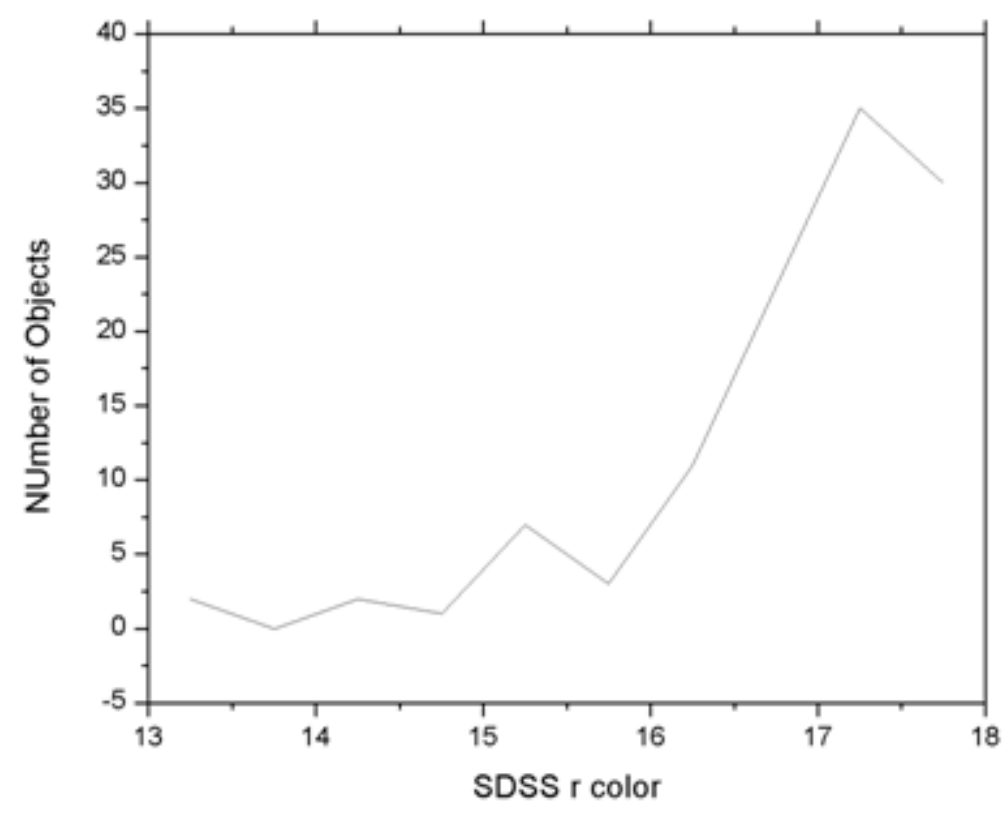

Figure 4. The number of ULIRGs/HLIRGs vs. SDSS $r$ magnitude. 


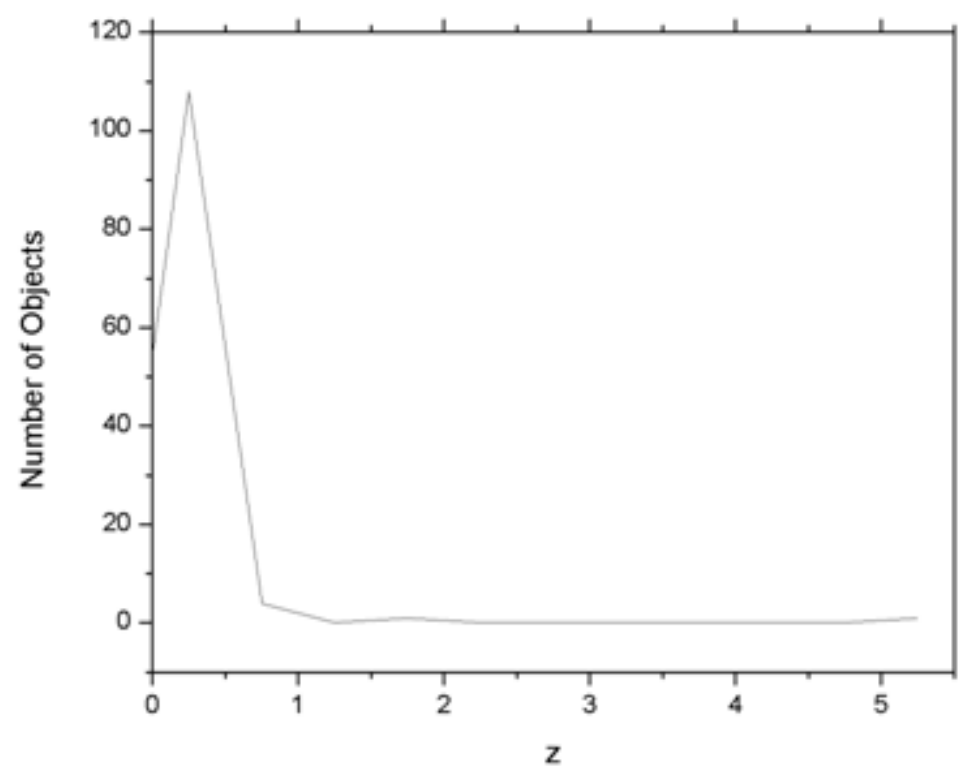

Figure 5. The number of ULIRGs/HLIRGs vs. redshift.

An extrapolation based on figures 4 and 5 shows the trend how many highluminosity IR galaxies might be expected at fainter magnitudes and higher redshifts.

To study the content of newly discovered ULIRGs, we plan to classify them by activity types from the SDSS spectra. These results will be given in our next paper.

\section{References}

Abrahamyan, H. V., Mickaelian, A. M., Knyazyan, A. V. 2015, Astronomy and Computing, 10, 99

Abolfathi, B., Aguado, D. S., Aguilar, G. et al. 2018, The Astrophysical Journal Supplement Series, 235, issue 2, article id. 42, p. 19

Cutri, R. M. et al. 2013, VizieR On-line Data Catalog: II/328. Originally published in: IPAC/Caltech

Duc, P.-A., Mirabel, I. F., Maza, J. 1997, A\&A Suppl., 124, 533

Ishihara, D., Onaka, T., Kataza, H., Salama, A., Alfageme, C., Cassatella, A. et al. 2010, $A \& A, 514,14$

Sanders, D. B., Mirabel, I. F. 1996, ARA\&A, 34, 749

Veilleux, S. 2002, Proceedings of IAU Colloquium 184, ASP Conference Series, 284, 111

Yamamura, I., Makiuti, S., Ikeda, N., Fukuda, Y., Oyabu, S., Koga, T., White, G. J., 2010,VizieR On-line Data Catalog: II/298. Originally published in: ISAS/JAXA 